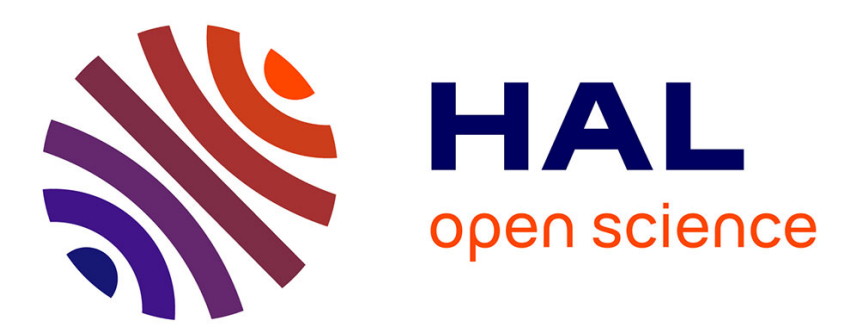

\title{
CONTRÔLE ACTIF DES INSTABILITÉS LIÉES AUX ÉCOULEMENTS. EXEMPLE DU POMPAGE DES SYSTĖMES DE COMPRESSION
}

M. Sebbani, M. Sunyach

\section{- To cite this version:}

M. Sebbani, M. Sunyach. CONTRÔLE ACTIF DES INSTABILITÉS LIÉES AUX ÉCOULEMENTS. EXEMPLE DU POMPAGE DES SYSTÈMES DE COMPRESSION. Journal de Physique IV Proceedings, 1992, 02 (C1), pp.C1-615-C1-618. 10.1051/jp4:19921132 . jpa-00251089

HAL Id: jpa-00251089

https://hal.science/jpa-00251089

Submitted on 1 Jan 1992

HAL is a multi-disciplinary open access archive for the deposit and dissemination of scientific research documents, whether they are published or not. The documents may come from teaching and research institutions in France or abroad, or from public or private research centers.
L'archive ouverte pluridisciplinaire HAL, est destinée au dépôt et à la diffusion de documents scientifiques de niveau recherche, publiés ou non, émanant des établissements d'enseignement et de recherche français ou étrangers, des laboratoires publics ou privés. 


\title{
CONTRÔLE ACTIF DES INSTABILITÉ LIÉES AUX ÉCOULEMENTS. EXEMPLE DU POMPAGE DES SYSTEMMES DE COMPRESSION
}

\author{
M. SEBBANI et M. SUNYACH \\ Laboratoire de Mécanique des Fluides et d'Acoustique, CNRS URA-263, Ecole Centrale de Lyon, \\ BP. 163, F-69131 Ecully cedex, France
}

\begin{abstract}
This paper describes an experimental study concerned with adaptive control of surge in compressor system. Attention is drawn to the control energy in particular.
\end{abstract}

\section{1 - INTRODUCTION}

Des travaux récents montrent que les instabilités d'écoulement qui sont initiées par un mécanisme linéaire sont sensibles au contrôle actif. Dans ce travail, nous nous sommes intéressés au contrôle autoadaptatif des systèmes faisant intervenir une compression sans propagation et plus particulièrement au pompage de systèmes de compression. Le système de contrôle est une contre réaction dont le rôle est de changer les caractéristiques de l'oscillateur, en particulier d'augmenter son amortissement.

\section{2 - PRINCTPE DU CONTROLEUR AUTO-ADAPTATIF}

Nous utilisons un algorithme temporel de minimisation reposant sur une méthode de gradient estimé. L'algorithme est schématisé en figure 1. La moyenne quadratique de l'erreur $\varepsilon$ (pression au microphone de contrôle) est minimisée par rapport aux coefficients variables du filtre A qui sont réactualisés suivant le schéma :

$$
A^{(n+1)}=A^{(n)}+2 k \varepsilon_{n} U_{n}
$$

$\operatorname{avec} \mathrm{u}_{\mathrm{n}}={ }^{\mathrm{t}} \mathrm{B} \mathrm{X}_{\mathrm{n}}$.

$B$ représente un filtre fixe modélisant le chemin secondaire (haut-parleur de contrôle-microphone de contrôle) introduit dans l'algorithme pour assurer la convergence. Le filtre principal A peut être selon les besoins à réponse impulsionnelle finie (RIF) ou infinie (RII). Cet algorithme est implanté en temps réel sur un contrôleur électronique équipé d'un microprocesseur spécialement réalisé pour cet usage.

Dans le cas du contrôle des oscillations de pompage, la détection se fait par mesure de la perturbation elle même, le système de contrôle fonctionne en boucle fermée et peut donc générer ses propres instabilités. La perturbation apportée par le contrôleur doit donc toujours rester inférieure à la perturbation naturelle.

\section{3 - MODELE ADOPTE}

Le pompage dans un système de compression est une oscillation auto-entretenue due à l'interaction entre le compresseur et son environnement (circuit récepteur). Il est caractérisé par une oscillation axiale du débit avec des amplitudes importantes susceptibles de provoquer des détériorations du système.

L'ensemble du système peut être schématisé par un conduit où le compresseur crée une discontinuité locale des grandeurs aérodynamiques, puis un réservoir de grande capacité et enfin une vanne de sortie qui absorbe l'énergie du compresseur. 
Pour la modélisation, on admet que dans la capacité les grandeurs thermodynamiques varient d'une façon isentropique et que la caractéristique du compresseur est quasi-stationnaire. D'autre part la vitesse est tellement faible dans la capacité que la pression est constante dans tout le volume. Dans les conduits caractérisés par leur faible volume, le fluide est supposé incompressible et son débit constant.

La structure du système de contrôle adoptée pour notre expérience est représentée par la figure 2 . Le contrôle des oscillations associées au pompage est réalisé par une modification active du volume de la capacité. Pour représenter l'action du contrôleur nous supposons le déplacement $\xi$ de la surface mobile (membrane du haut-parleur) proportionnel aux fluctuations de pression $(\delta \psi)$ dans la capacité [1] :

$$
\xi=\mathrm{Z} \delta \psi
$$

Les étapes conduisant à établir les équations régissant la dynamique de ce système de compression sont bien connues [2]. En prenant en compte le débit supplémentaire introduit par le contrôle, les équations linéarisées de quantité de mouvement dans les conduits compresseur et vanne, en négligeant l'inertie de la vanne, et celle de la conservation de la masse dans la capacité conduisent à l'équation caractéristique du système qui s'écrit :

$$
\begin{aligned}
& \mathrm{s}^{2}+\left[\frac{1}{\mathrm{BT}^{\prime}(1+\widetilde{\mathrm{Z}})}-\left(\mathrm{B} \psi^{\prime}-\mu\right)\right] \mathrm{s}+\frac{1}{(1+\widetilde{\mathrm{Z}})}\left[1-\frac{\left(\mathrm{B} \psi^{\prime}-\mu\right)}{\mathrm{BT} T^{\prime}}\right]=0 \\
& \text { où } \quad B=\frac{U}{2 \omega_{\mathrm{H}} \mathrm{L}}: \quad \text { avec } U \text { vitesse périphérique, } \omega_{\mathrm{H}} \text { la fréquence de résonance } \\
& \text { de Helmholtz et } L \text { longueur effective de la conduite compresseur, } \\
& \text { - } \psi^{\prime} \text { et } T^{\prime} \text { : } \\
& \text { les dérivées des caractéristiques pression-débit, compresseur }\left(\psi\left(\phi_{c}\right)\right) \\
& \text { et vanne }\left(T\left(\phi_{V}\right)\right) \text { au point de fonctionnement, } \\
& \begin{array}{lll}
\text { - } & \mu & \text { représente les pertes visqueuses dans la conduite compresseur } \\
& \widetilde{Z}=X+\widetilde{Z}_{\xi}: & \text { la loi de contrôle où } \widetilde{Z}_{\xi} \text { représente la force de contrôle au moyen }
\end{array} \\
& \text { duquel nous désirons améliorer la stabilité du système et } X \text { lié au } \\
& \text { mouvement de la surface mobile dû à la pression instable dans la } \\
& \text { capacité. }
\end{aligned}
$$

La résolution de cette équation dont la solution est de la forme $s=\beta+j \omega$ et en prenant $\beta=0$ (taux de croissance nul) nous donne l'équation suivante définissant la limite de stabilité du système :

$$
B \psi^{\prime}-\mu-\frac{\omega^{2}+\left(B \psi^{\prime}-\mu\right)^{2}}{B T^{\prime}}+\widetilde{Z}_{i} \omega\left[\omega^{2}+\left(B \psi^{\prime}-\mu\right)^{2}\right]=0
$$

où $\widetilde{Z}_{\mathbf{i}}$ est la partie imaginaire de $\widetilde{Z}_{\xi}$.

\section{4 -ASPECT ENERGETIQUE}

Le mécanisme par lequel le contrôleur supprime les oscillations dans le système de compression peut être interprété en considérant l'énergie du système. L'excédent d'énergie contribuant à l'accroissement des perturbations est donné par:

$$
\Delta \mathrm{E}=\mathrm{E}_{\mathrm{C}}-\mathrm{E}_{\mathrm{V}}-\mathrm{E}_{\xi}
$$

où $\quad \mathrm{E}_{\mathrm{C}}$ : est l'énergie produite sur un cycle par le compresseur après dissipation d'énergie dans sa conduite.

$\mathrm{E}_{\mathrm{V}}$ : énergie dissipée dans la vanne

$E_{\xi}: \quad$ énergie absorbée par le contrôleur.

Les calculs effectués [3] en utilisant les équations régissant le système citées ci-dessus, montrent que 
l'énergie du contrôleur dépend de l'amplitude des perturbations plutôt que de l'amplitude du compresseur à l'état stable, ceci montre qu'il peut y avoir une grande différence entre l'énergie du contrôleur et l'énergie moyenne du compresseur; le contrôleur ne consommant qu'une faible partie de celle-ci.

La stabilité du système de compression est déterminée par le signe du gain d'énergie $\Delta \mathrm{E}$. Pour $\Delta \mathrm{E}>0$, les perturbations reçoivent continuellement de l'énergie et leur amplitude s'accroît et par suite le système est instable. Pour $\Delta \mathrm{E}<0$, l'énergie est dissipée vers l'extérieur du système dans lequel les perturbations s'amortissent et le système devient stable. La limite de stabilité est obtenue en prenant $\beta=0$ et $\Delta E=0$. L'équation définissant la limite de stabilité est alors identique à l'équation (4) obtenue précédemment.

\section{5 - RESULTATS}

Le système de compression utilisé dans notre expérience est formé d'un ventilateur axial double étage (type ETRI $86 \mathrm{CD}$ ) de vitesse $2850 \mathrm{tr} / \mathrm{mn}$. La conduite compresseur de longueur $\mathrm{L}=0,20 \mathrm{~m}$ a une sec-

tion interne $\mathrm{A}_{C}=0,0153 \mathrm{~m}^{2}$ et un coefficient de perte $\mu=0,4$. L'écoulement produit par le ventilateur

alimente une large capacité de volume $\mathrm{V}=0,7 \mathrm{~m}^{3}$. La décharge de cette capacité s'effectue à travers une vanne de sortie de diamètre très petit comparé aux dimensions de la capacité. La fréquence de résonance de Helmholtz mesurée est de $8,9 \mathrm{~Hz}$.

De la connaissance de ces valeurs des différents paramètres et des caractéristiques compresseur et vanne, nous avons déterminé (figure 3) la carte de stabilité dans le plan gain-phase définie par l'équation (4). Cette carte est similaire pour tous les systèmes instables modélisés par une équation du second ordre. On la retrouve par exemple dans Candel et al. [4] pour le cas des instabilités de combustion. On observe sur cette carte que lorsque $B$ augmente, la région stable se rétrécit de plus en plus et le contrôle devient plus difficile. Il en est de même lorsque la pente de la caractéristique compresseur $\psi^{\prime}$ devient plus raide.

Pour examiner l'influence du contrôle on compare le système de compression avec et sans contrôle, lorsque le compresseur est en pompage le contrôleur supprime en 3 ou 4 cycles l'instabilité (figure 4); l'amplitude des fluctuations de pression et de débit est nettement atténuée et le cycle de pompage est réduit à un point de fonctionnement stable. On observe également ceci sur le spectre du signal du microphone où les pics à la fréquence fondamentale de l'instabilité et ses harmoniques sont réduits totalement jusqu'au fond du spectre (figure 5). Dans le cas étudié on constate que le contrôle est efficace quelque soit la position du point de fonctionnement sur la caractéristique du compresseur et qu'il reste efficace même lorsque le cycle de pompage est déjà développé.

\section{6 - CONCLUSION}

Les résultats reportés ici ont permis de valider l'utilisation des algorithmes temporels type gradient pour la mise en oeuvre de contrôle actif adaptatif des instabilités d'écoulement. L'analyse énergétique effectuée montre que l'énergie, nécessaire à l'action du contrôle, est petite et ne dépend pas de l'énergie statique produite par le compresseur. L'application industrielle de ce moyen de contrôle, sur les systèmes présentant des hauts niveaux d'énergie, est donc envisageable au moins pour compenser une instabilité naissante et aussi pour détecter son apparition en fonction de l'activité du contrôleur.

\section{REFERENCES}

[1] A.H. EPSTEIN, J.E. FFOWCS WILLIAMS \& E.M. GREITZER, "Active suppression of compressor instabilities", AIAA-86-1994.

[2] E.M. GREITZER, "Surge and rotating stall in axial flow compressors", Trans. ASME, Journal of Engineering for Power, Vol. 98, April 1976, pp. 190-217.

[3] M. SEBBANI, "Contrôle actif des couplages aéroacoustiques à l'aide de systèmes autoadaptatifs", Thèse de Doctorat, Ecole Centrale de Lyon, $n^{\circ}$ 91-18, juin 1991.

[4] S.M. CANDEL \& T.J. POINSOT, "Interaction between acoustics and combustion", Proc. of I.O.A., Vol. 10, part 2, pp. 103-153. 

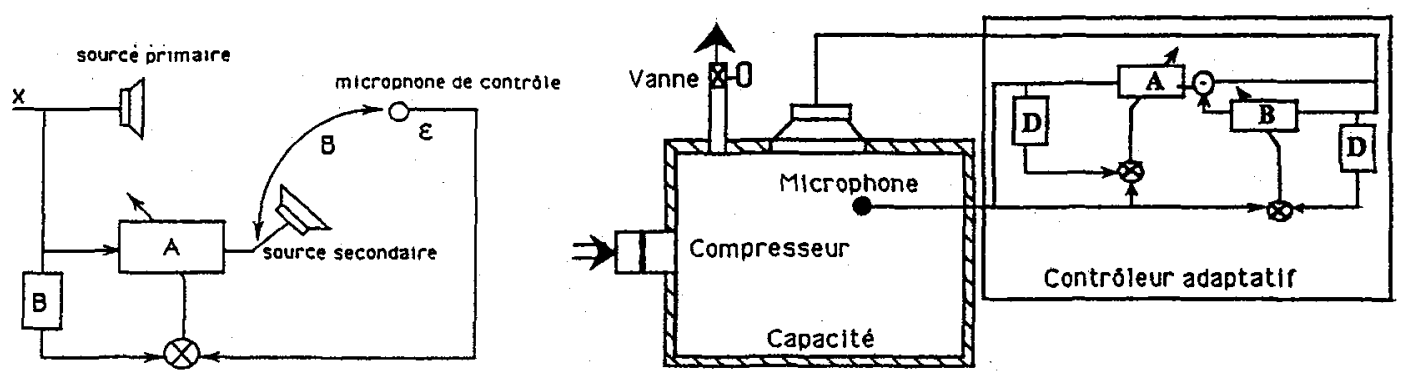

Fig. 1 : Schéma de l'algorithme de gradient

Fig. 2 : Contrôle du pompage (fittre RII)

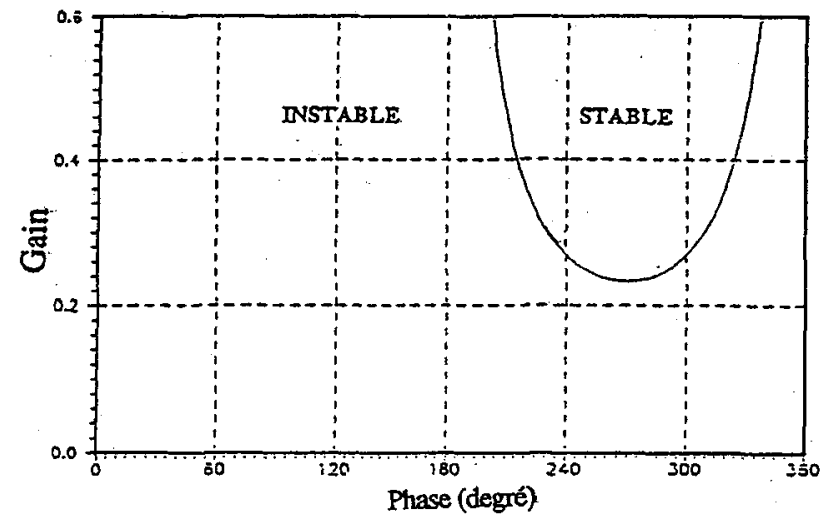

Fig. 3 : Carte de stabilité $(B=0,57)$

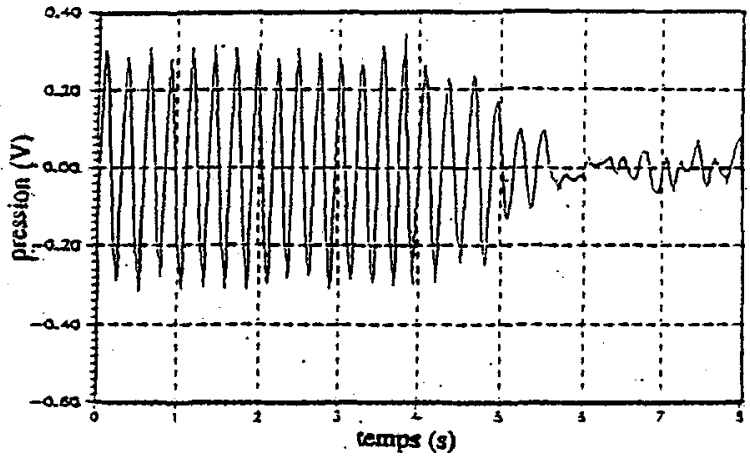

Fig. 4 : Suppression des fluctuations de pression

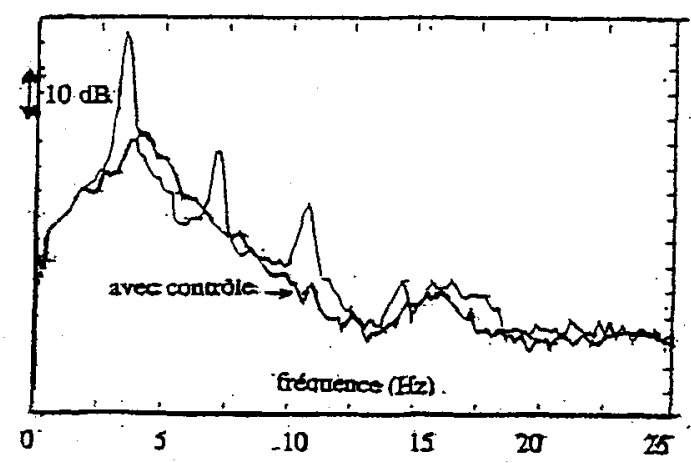

Fig. 5 : Spectre des fluctuations de pression avec et sans controle 